\title{
Poor COVID-19 Preventive Practice among Healthcare Workers in Northwest Ethiopia, 2020
}

\author{
Belayneh Ayanaw Kassie $\mathbb{D}^{1},{ }^{1}$ Aynishet Adane, ${ }^{2}$ Eskeziaw Abebe Kassahun $\left(\mathbb{D},{ }^{3}\right.$ \\ Amare Simegn Ayele, ${ }^{4}$ and Aysheshim Kassahun Belew ${ }^{5}$ \\ ${ }^{1}$ Department of Women's and Family Health, School of Midwifery, College of Medicine and Health Sciences, \\ University of Gondar, Gondar, Ethiopia \\ ${ }^{2}$ Department of Internal Medicine, School of Medicine, College of Medicine and Health Sciences, University of Gondar, \\ Gondar, Ethiopia \\ ${ }^{3}$ Department of Midwifery, College of Health Sciences, Woldia University, Woldia, Ethiopia \\ ${ }^{4}$ Department of Midwifery, College of Medicine and Health Sciences, Debre Tabor University, Debre Tabor, Ethiopia \\ ${ }^{5}$ Department of Human Nutrition, Institute of Public Health, University of Gondar, Gondar, Ethiopia
}

Correspondence should be addressed to Belayneh Ayanaw Kassie; belyuayanaw@gmail.com

Received 20 June 2020; Revised 2 October 2020; Accepted 4 October 2020; Published 20 October 2020

Academic Editor: Hamidreza Karimi-Sari

Copyright (C) 2020 Belayneh Ayanaw Kassie et al. This is an open access article distributed under the Creative Commons Attribution License, which permits unrestricted use, distribution, and reproduction in any medium, provided the original work is properly cited.

\begin{abstract}
Background. The novel coronavirus disease (COVID-19) pandemic outbreak affects the global social, economic, and political context and becomes a significant threat to healthcare providers who are among the exposed groups to acquire and transmit the disease while caring and treating patients. It is crucial to comply with prevention recommendations so as to stay safe and protected. Therefore, this study aimed to assess COVID-19 preventive practice and associated factors among healthcare workers in Northwest Ethiopia. Methods. An institution-based cross-sectional study was conducted among 630 healthcare workers in Northwest Ethiopia from March to April 2020. A multistage sampling technique was used to select study participants. A pretested and structured self-administered questionnaire was used to collect data. The data were entered using Epi Info 7 and analyzed using STATA 16 statistical software. Both bivariate and multivariable logistic regression analyses were employed to identify associated factors. Adjusted odds ratio (AOR) with 95\% confidence interval was used to determine independent predictors of COVID-19 preventive practice. In multivariable analysis, a variable with a $P$ value of less than 0.05 was considered as statically significant. Result. Among 630 healthcare workers participated in the study, the overall good preventive practice towards COVID-19 was found to be $38.73 \%$ (95\% CI: 34.8, 42.5). Being a male healthcare provider (AOR $=1.48$; 95\% CI: 1.02, 2.10), having work experience of $6-10$ years $(\mathrm{AOR}=2.22 ; 95 \% \mathrm{CI}: 1.23,4.00)$, and having poor attitude towards COVID-19 (AOR $=2.22 ; 95 \% \mathrm{CI}$ : $1.03,2.22)$ were found to be significantly associated with poor COVID-19 preventive practice among healthcare workers. Conclusion. Overall compliance towards COVID-19 preventive practice among healthcare workers was found to be low. Multiple education and training platforms with focus on COVID-19 preventive measures and adequate personal protective equipment and supplies should be provided for healthcare providers.
\end{abstract}

\section{Introduction}

The novel coronavirus disease (COVID-19), defined as an illness caused by severe acute respiratory syndrome coronavirus 2 (SARS-Cov-2), was first identified as an outbreak of respiratory illness cases and first confirmed in December 2019 in Wuhan, Hubei Province, China $[1,2]$. Astonishingly, in the first three months after COVID-19 emerged, a devastating number of new cases were reported across China and several countries around the world [3], and it was finally declared as a global pandemic by the World Health Organization (WHO) on March 2020 [4]. Evidence indicates that COVID-19 transmitted through respiratory droplets via contact routes such as the mouth, nose, and conjunctiva or eyes $[5,6]$. 
According to the Worldometer report, the outbreak has been confirmed in over 21,628,638 individuals worldwide and resulted in more than 769,128 deaths as of August 16, 2020 , of which $99 \%$ cases were found in mild condition. More than 213 countries reported laboratory-confirmed coronavirus cases. In Africa, 1,113,246 confirmed cases and 25,385 deaths were reported. Moreover, in Ethiopia, 28,894 confirmed cases and 509 deaths were reported [7].

Even though the outbreak is a global pandemic, it is important to note that the problem needs more attention in Africa because the African countries have limited healthcare system capacity to control the pandemic [8]. Measures to prevent transmission in healthcare settings are an immediate priority to slowdown the demand for specialized healthcare such as intensive care unit beds, safe guarding risk groups, protecting healthcare workers, and minimizing the export of the cases to other healthcare facilities and the wider community [9].

Healthcare workers (HCWs) are at a high risk of exposure to COVID-19 due to their direct contact with patients, so the triage of the patient with acute respiratory symptoms should be determined, the contact distance should be arranged to be at least 2 meters, and patients should wear face masks. During the care of these patients, the HCW should wear necessary personal protective equipment (PPE) and keep hand hygiene [10]. Thus, poor WASH (Water, Sanitation, and Hygiene) and infection prevention and control (IPC) lead to hospital-acquired infections and transmission of disease from health facilities to the community that will exacerbate the outbreak and spread of infections [6].

A Cochrane trusted evidence showed that long and constantly changing local guidelines, low level of support by the management, lack of enough space for isolation, lack of quality and adequate PPE, and fear of the patients being stigmatized if mask is worn are important impeding factors to follow the IPC guidelines by the healthcare workers [11].

Ultimately, HCWs play a pivotal role in averting acquired infections from hospitals via practicing effective and evidence-based infection prevention and control strategies [12]. However, despite limited data available on practice compliance of HCWs for other infectious diseases [13], no data are available on practice compliance of HCWs against the predefined COVID-19 preventive measures in the study area and the country at large. Therefore, the study will be the first of its kind in informing the healthcare taskforce about the status of HCWs in preventing COVID-19 and an immediate action will be taken. HCWs will take an alarming precaution to protect themselves, as well as the community as a whole.

\section{Methods}

2.1. Study Design and Setting. This institution-based crosssectional study was conducted from March to April 2020 in Northwest Ethiopia, Central and South Gondar zones of the Amhara regional state, to explore the preventive practice of healthcare providers towards COVID-19. The administrative zones have an estimated population of $5,137,443$, and there are 18 public hospitals and 181 public health centers (North Gondar: 10 hospitals and 85 health centers; South Gondar: 8 hospital and 96 health centers).

2.2. Sampling Strategy. Sample size was calculated using the single population proportion formula by considering a $95 \%$ confidence interval (CI), $5 \%$ margin of error $(d)$, proportion $(P) 50 \%$, and 1.5 of design effect. Considering $10 \%$ of nonresponse rate, the final sample was 634 HCWs. A multistage sampling technique was used to select participants. From the total 18 hospitals and 181 health centers, 5 hospitals and 20 health centers were selected using simple random sampling. Then, based on the number of HCWs in each facility, the total sample was proportionally allocated to each facility, and then, each study participant was selected from the list of HCWs in each respective facility using simple random sampling.

2.3. Data Collection. The self-reported questionnaire was developed through reviewing different literatures that included sociodemographic characteristics such as age, sex, ethnicity, religion, marital status, types of working facility and level of education, knowledge questions on the clinical presentations and transmission routes, attitude of HCWs, and preventive practice towards COVID-19 outbreak. A pretest was conducted among providers out of the study area. Training was provided on data collection, and necessary COVID-19 precautions were provided during data collection. Six BSc public health officers and two master of public health holders collected data and supervised data collection, respectively.

2.4. Measurements. The dependent variable is preventive practice towards COVID-19. Preventive practices of HCWs were measured by 5 practice-related questions (Table 1). A correct answer was assigned 1 point, and an incorrect/unknown answer was assigned 0 points. The total score ranged from 0 to 5 , with a higher score based on the mean value ( $\geq 3.13$ ) denoting a "good preventive practice" of COVID-19.

Attitudes towards COVID-19 were measured by six questions (Table 2) about the agreement on the final control and confidence in winning the battle against COVID-19. A correct answer was assigned 1 point, and an incorrect/unknown answer was assigned 0 points. The total attitude score ranged from 0 to 6 , with a score above the mean value ( $\geq 4.02)$ denoting a "good attitude" towards COVID-19.

The COVID-19 knowledge of HCWs was assessed by using 15 questions (Table 2). A correct answer was assigned 1 point, and an incorrect/unknown answer was assigned 0 points. The total knowledge score ranged from 6 to 15 , with a higher mean score $(\geq 13.56)$ denoting a "good knowledge" of COVID-19.

2.5. Statistical Analysis. Data were entered into Epi Info 7 and analyzed using STATA 16. Descriptive statistics were used to describe participants' sociodemographics and response of knowledge, attitude, and preventive practice 
TABLE 1: Preventive practice of healthcare professions towards COVID-19.

\begin{tabular}{lrr}
\hline Preventive practice questions & Yes (\%) & Response \\
\hline In the past 4 weeks, had travel history out of my town & $536(85.08)$ & No (\%) \\
In the past 4 weeks, had been in any crowded place & $273(43.33)$ & $94(14.92)$ \\
In the past 4 weeks, had consistently used face mask in the working place & $228(36.19)$ & $357(56.67)$ \\
In the past 4 weeks, had shaken hands with friends/staff & $404(64.13)$ & $402(63.81)$ \\
In the past 4 weeks, had washed hands frequently with soap/used sanitizers & $529(83.97)$ & $226(35.87)$ \\
\hline
\end{tabular}

Note. Good preventive practice if the mean value $\geq 3.13$.

Table 2: Descriptive statistics of knowledge and attitude of HCWs towards COVID-19.

\begin{tabular}{|c|c|c|}
\hline \multirow{2}{*}{ Knowledge questions } & \multicolumn{2}{|c|}{ Response } \\
\hline & Yes $(\%)$ & No $(\%)$ \\
\hline Fever is one of the possible typical symptoms of COVID-19 & $610(96.83)$ & $20(3.17)$ \\
\hline Dry cough is the possible typical symptom of COVID-19 & $559(88.73)$ & $71(11.27)$ \\
\hline Fatigue is the possible typical symptom of COVID-19 & $467(74.13)$ & $163(25.87)$ \\
\hline Myalgia is the possible typical symptom of COVID-19 is & $410(65.08)$ & $220(34.92)$ \\
\hline Hand washing and/or sanitizer use frequently can help to protect you from COVID-19 & $611(96.98)$ & $19(3.02)$ \\
\hline Covering the nose with hands during sneezing can help to protect you from COVID-19 & $545(86.51)$ & $85(13.49)$ \\
\hline Wearing a face mask can help to protect you from COVID-19 & $552(87.62)$ & $78(12.38)$ \\
\hline Keeping physical distance can help to protect you from COVID-19 & $569(90.32)$ & $61(9.68)$ \\
\hline Asymptomatic persons with COVID-19 can transmit the disease & $611(96.98)$ & $19(3.02)$ \\
\hline The isolation period for a COVID-19 suspect case is 2 weeks & $615(97.62)$ & $15(2.38)$ \\
\hline Risk of death from COVID-19 is higher among patients with an underlying chronic illness & $574(91.11)$ & $56(8.89)$ \\
\hline Healthcare workers are at higher risk for COVID-19 infection & $620(98.41)$ & $10(1.59)$ \\
\hline Covid-19 is transmitted via respiratory droplets & $611(97.98)$ & $19(3.02)$ \\
\hline There are specific vaccines to prevent COVID-19 as of today & $589(93.49)$ & $41(6.51)$ \\
\hline Antibiotics are effective in treating COVID-19 & $603(95.71)$ & $27(4.29)$ \\
\hline Overall having good knowledge & $404(64.13)$ & $226(35.87)$ \\
\hline \multicolumn{3}{|l|}{ Attitude questions } \\
\hline Believe that COVID-19 will finally be successfully controlled & $437(69.37)$ & $193(30.63)$ \\
\hline Hand hygiene is important in controlling the spread COVID-19 & $603(95.71)$ & $27(4.29)$ \\
\hline Wearing masks is important in controlling the spread COVID-19 & $597(94.76)$ & $33(5.24)$ \\
\hline Think you may probably get infected with COVID-19 & $445(70.63)$ & $185(29.37)$ \\
\hline Had confidence that Ethiopia can win the battle against COVID-19 & $347(55.08)$ & $283(44.92)$ \\
\hline Think the government of Ethiopia is doing enough to prevent and control COVID-19 outbreak & $105(16.67)$ & $525(83.33)$ \\
\hline Overall good attitude & $445(70.63)$ & $185(29.37)$ \\
\hline
\end{tabular}

Note. Good knowledge if the mean value $\geq 13.56$, and good attitude if the mean value $\geq 4.02$.

questions. Cronbach's alpha coefficient of the knowledge questionnaire was checked. Age of the respondents, knowledge, attitude, and preventive practice data were reported as mean \pm SD. A binary logistic regression model was fitted to identify the associated factors. Then, variables with a $P$ value less than 0.2 were fitted to multivariable analysis. The goodness of fit was checked by using the Hosmer-Lemeshow goodness of fit. Multivariate logistic regression analysis with the enter method was used to identify predictors of preventive practice of COVID-19. A $P$ value less than 0.05 was considered as statistically significant.

\section{Results}

3.1. Sociodemographic Characteristics of Study Participants. A total of $630 \mathrm{HCWs}$ participated in the study, with the response rate of $99.2 \%$. Of these, 395 (62.7\%) were male providers. The mean age of participants was $31.2 \pm 7$ years. More than one-third of the participants were working in the health center. A highest proportion of respondents were found to be 20-30 years old (62.22\%), have a bachelor's degree (BSc) in their current level of education (56.35), were Orthodox Christian followers (92.38\%), and have one to five years of work experience (51.11\%) (Table 3).

\subsection{Participants' Knowledge and Attitude towards COVID-19.} Almost all 621 (98.6\%) ever heard about COVID-19 virus. The most common source of information was news from television and radio. About two-thirds (67.3\%) of the respondents reported that they use social media (Facebook, Twitter, WhatsApp, YouTube, and Instagram) to obtain information about COVID-19 (Figure 1).

Table 2 presents the response of the respondent's knowledge and attitude. HCWs were generally knowledgeable, and almost $64.1 \%$ of the participants had good knowledge on the cause, clinical symptoms, and prevention mechanisms. Moreover, 445 (70.63\%) respondents had positive attitude towards COVID-19 (Table 2). 
TABLE 3: Sociodemographic characteristics of HCWs in Northwest Ethiopia, $2020(n=630)$.

\begin{tabular}{|c|c|c|}
\hline Variables & Number $(N)$ & Percentage (\%) \\
\hline $\begin{array}{l}\text { Age of study participants } \\
20-30 \\
31-40 \\
\geq 41\end{array}$ & $\begin{array}{c}392 \\
180 \\
58 \\
\end{array}$ & $\begin{array}{c}62.22 \\
28.57 \\
9.21 \\
\end{array}$ \\
\hline $\begin{array}{l}\text { Type of working health facility } \\
\text { Health center } \\
\text { Hospital }\end{array}$ & $\begin{array}{l}210 \\
420 \\
\end{array}$ & $\begin{array}{l}33.33 \\
66.67 \\
\end{array}$ \\
\hline $\begin{array}{l}\text { Level of education } \\
\text { Diploma } \\
\text { BSc } \\
\text { MSc } \\
\text { Physician }\end{array}$ & $\begin{array}{c}116 \\
355 \\
53 \\
106\end{array}$ & $\begin{array}{c}18.41 \\
56.35 \\
8.41 \\
16.83\end{array}$ \\
\hline $\begin{array}{l}\text { Profession } \\
\text { Nurse } \\
\text { Midwife } \\
\text { Physician } \\
\text { Laboratory technician } \\
\text { Pharmacist } \\
\text { Anesthetist } \\
\text { Health officer } \\
\text { Radiography } \\
\end{array}$ & $\begin{array}{c}245 \\
120 \\
102 \\
69 \\
41 \\
16 \\
29 \\
8 \\
\end{array}$ & $\begin{array}{c}38.90 \\
19.0 \\
16.20 \\
11.0 \\
6.50 \\
2.50 \\
4.60 \\
1.30 \\
\end{array}$ \\
\hline $\begin{array}{l}\text { Ethnicity } \\
\text { Amhara } \\
\text { Qimant } \\
\text { Oromo } \\
\text { Others }\end{array}$ & $\begin{array}{l}574 \\
26 \\
17 \\
13\end{array}$ & $\begin{array}{c}91.11 \\
4.13 \\
2.70 \\
2.06\end{array}$ \\
\hline $\begin{array}{l}\text { Religion } \\
\text { Muslim } \\
\text { Orthodox Christian } \\
\text { Protestant }\end{array}$ & $\begin{array}{c}33 \\
582 \\
15 \\
\end{array}$ & $\begin{array}{c}5.24 \\
92.38 \\
2.38 \\
\end{array}$ \\
\hline $\begin{array}{l}\text { Year of experience } \\
1-5 \text { years } \\
6-10 \text { years } \\
\geq 11 \text { years }\end{array}$ & $\begin{array}{l}322 \\
174 \\
134\end{array}$ & $\begin{array}{l}51.11 \\
27.62 \\
21.27\end{array}$ \\
\hline
\end{tabular}

3.3. Preventive Practice towards COVID-19. The overall COVID-19 preventive practice among HCWs found was to be $244(38.7 \%)(95 \% \mathrm{CI} ; 34.8,42.5 \%)$. In the previous 4 weeks, about $84 \%$ of the participants washed their hands frequently with soap/hand sanitizers before and after contacting patients and entering their home and $36.3 \%$ of healthcare providers were not using face mask in the working place (Table 1).

\subsection{Factors Associated with HCW' COVID-19 Preventive} Practice. Both bivariate and multivariable logistic regression analyses were used to see the effect of those variables on preventive practice towards COVID-19. In bivariate logistic regression analysis, variables such as sex, age, level of education, years of working experience, and attitudes of HCWs were associated with COVID-19 preventive practice. After controlling for the effects of potentially confounding variables using multivariate logistic regression sex, years of working experience and attitude were found to be significantly associated with poor COVID-19 preventive practice.
In comparison with female, male HCW s had higher odds of having poor preventive practice $(\mathrm{AOR}=1.48 ; 95 \% \mathrm{CI}$ : $1.02,2.10)$. The odds of having poor COVID-19 preventive practice was 2.22 times higher among HCWs with 6-10 years working experience $(\mathrm{AOR}=2.22 ; 95 \% \mathrm{CI}: 1.23,4.00)$ as compared to 11 or more years of working experiences. Moreover, HCWs having poor attitude towards COVID-19 virus were 1.51 times more likely $(\mathrm{AOR}=2.22$; $95 \% \mathrm{CI}$ : 1.03 , 2.22) to have poor preventive practice as compared to those having good attitude (Table 4).

\section{Discussion}

HCWs are at significant risk of COVID-19 infection during patient care delivery [14]. As consistent application of prevention mechanisms, use of PPE can diminish the risk of infection for healthcare workers [11]. This study assessed HCWs' compliance with the recommendations of COVID-19 preventive practice in Northwest Ethiopia.

The finding of this study showed that the overall good COVID-19 preventive practice among HCPs was found to be $38.7 \%$ (95\% CI: 34.8, 42.7). This finding was lower than that of a study conducted among HCWs in China (87\%) [15], and Uganda (74\%) [16]. The possible explanation for the disparity might be due to the difference in the economic status of the country, which increases the capacity and distribution of protective equipment of the healthcare system. Moreover, the compliance towards COVID-19 prevention recommendations among those HCWs could be due to differences in measurement, country context, and disease burden. In the Uganda study, all samples were taken from a single facility.

In this study, only $36.1 \%$ reported consistent use of face masks in the workplace which significantly facilitate the chance to acquire and transmit the disease. This could be because some HCPs may find it difficult to use masks and other equipment when it made patients feel isolated, frightened, or stigmatized and some HCWs even found masks uncomfortable to use [11]. Even though the WHO and other partners announced there will be a global shortage of PPE due to increased demand [17], there are suggestions that facemasks may be used intermittently or continuously for around eight hours [18]. Adverse effects of facemasks increase with more than eight hours of use [19]; however, in this study, the median duration of using a single face mask was found to be 6 days, which contradicts with evidence that pathogens may be present on the outer surface of around $10 \%$ masks and risks increase with prolonged mask use [20].

Moreover, $84 \%$ of HCPs had practiced frequent hand washing with soap and water or using sanitizer before and after contacting patients and entering their home. This could be a good practice in the context of a country with limited resources. However, in the recent 4 weeks prior to data collection, $43.3 \%$ providers reported that they had been in a crowded place as in churches, markets, and funeral ceremonies and $64.1 \%$ had shaken hands with staff, friends, or other people. This could be due to the influences from cultural and social intimacy in the community and being absent from such social and cultural events might cause misunderstanding and get stigma from the community. 


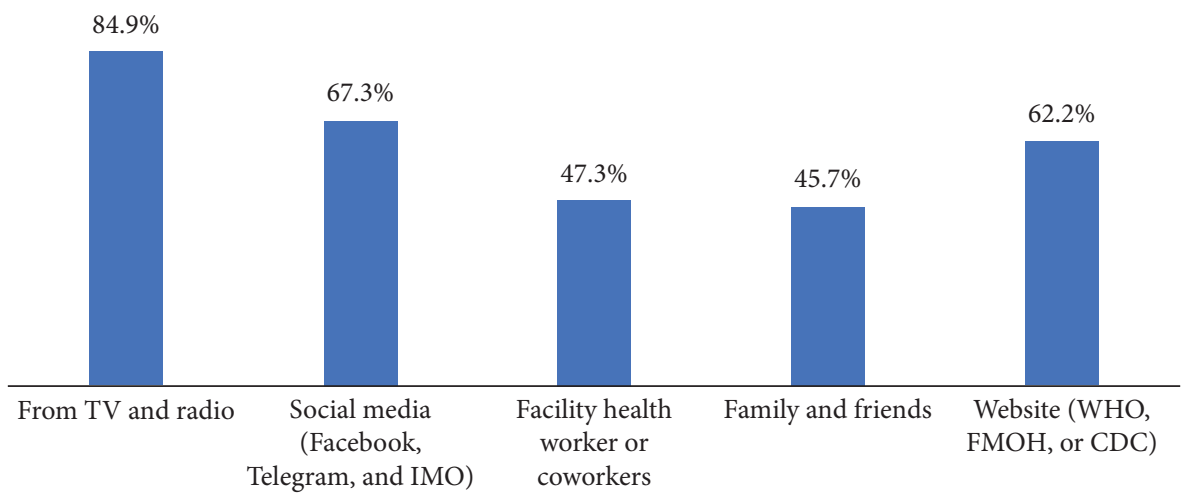

FIGURE 1: Source of information about COVID-19 virus.

TABLE 4: Bivariate and multivariate logistic regression analysis for factors associated with poor COVID-19 preventive practice among HCWs in Northwest Ethiopia, 2020.

\begin{tabular}{|c|c|c|c|c|c|}
\hline \multirow{2}{*}{ Variables } & \multicolumn{2}{|c|}{ Poor preventive practice } & \multirow{2}{*}{ COR (95\% CI) } & \multirow{2}{*}{ AOR (95\% CI) } & \multirow{2}{*}{$P$ value } \\
\hline & Yes & No & & & \\
\hline \multicolumn{6}{|l|}{ Age } \\
\hline $20-30$ & $273(43.33)$ & 119 (18.89) & $2.63(1.51,4.61)$ & $1.46(0.65,3.28)$ & 0.357 \\
\hline $31-40$ & $86(13.65)$ & $94(14.92)$ & $1.05(0.58,1.90)$ & $0.72(0.35,1.45)$ & 0.355 \\
\hline 41 & $27(4.29)$ & $31(4.92)$ & 1.0 & & \\
\hline \multicolumn{6}{|l|}{$\operatorname{Sex}$} \\
\hline Female & $133(21.11)$ & $102(16.19)$ & 1.0 & 1.0 & \\
\hline Male & $253(40.16)$ & $142(22.54)$ & $1.37(0.98,1.90)$ & $1.48(1.02,2.10)$ & 0.031 \\
\hline \multicolumn{6}{|c|}{ Level of education } \\
\hline Diploma & $60(9.5)$ & $56(8.9)$ & $0.53(0.31,0.91)$ & $0.68(0.38,1.28)$ & 0.207 \\
\hline $\mathrm{BSC}$ & $228(36.2)$ & $127(20.2)$ & $0.89(0.56,1.40)$ & $1.05(0.64,1.72)$ & 0.846 \\
\hline MSc & $27(4.3)$ & $26(4.1)$ & $0.51(0.26,1.00)$ & $0.85(0.40,1.80)$ & 0.670 \\
\hline Physician & $71(11.3)$ & $35(5.6)$ & 1.0 & 1.0 & \\
\hline \multicolumn{6}{|c|}{ Year of working experience } \\
\hline $1-5$ years & $218(34.6)$ & $104(16.5)$ & $3.16(2.05,4.71)$ & $1.71(0.90,3.24)$ & 0.102 \\
\hline $6-10$ years & $114(18.1)$ & $60(9.5)$ & $2.82(1.77,4.48)$ & $2.22(1.23,4.00)$ & 0.008 \\
\hline$\geq 11$ years & $54(8.6)$ & $80(12.7)$ & 1.0 & 1.0 & \\
\hline \multicolumn{6}{|l|}{ Attitude } \\
\hline Poor & $130(20.6)$ & $55(8.7)$ & $1.75(1.21,2.52)$ & $1.51(1.03,2.22)$ & 0.035 \\
\hline Good & $256(40.6)$ & $189(30.0)$ & 1.0 & 1.0 & \\
\hline
\end{tabular}

In this study, compliance towards COVID-19 prevention was significantly affected by sex. Female HCWs were found to better comply with prevention recommendations. This finding is consistent with that of a community-based study conducted among Chinese residents [21] that female participants implemented preventive practice than males. Moreover, in another study in Malaysia, females were found to better practice hand washing than males [22]. This could be due to the women's responsibility in taking care of children and family in the Ethiopian culture, which may influence them to fear and comply with prevention recommendations.

HCPs having 6-10 years of experience had reported better compliance with COVID-19 prevention recommendations than providers with 11 or more years of experience. This finding is also supported by a study conducted among the Malaysian community [22] that those older people with the age of greater than 50 years were found to be less likely to wear facemasks. This could be because as the HCPs age and experience increases, the exposure and fear for such outbreaks will reduce and may tend to normalize things as normal.

Attitude towards COVID-19 transmission, prevention, and control was among the factors that showed a significant association with compliance, and those providers who had poor attitude towards the disease, its transmission, and controlling movements were less likely to comply with the COVID-19 prevention recommendations. This finding was in line with studies conducted among communities in China [21] that those who were hopeful and confident that the disease will finally be controlled successfully were more likely to implement preventive practices.

\section{Conclusions}

Multiple education and training platforms with a focus on COVID-19 preventive measures need to be provided for HCPs. As a risk group for acquiring and transmitting the 
diseases and the absence of any vaccine or treatment as of today, HCPs should comply with COVID-19 preventive measures and should rely on infection prevention and patient safety measures. A lack of or poor quality PPE is a serious concern for healthcare providers [11]. This would contribute to the reduced utilization of facemasks in the work place, which requires the need to avail the required protective supplies.

\section{Abbreviations}

AOR: $\quad$ Adjusted odds ratio

CDC: $\quad$ Centers for Disease Control and Prevention

COVID-19: Coronavirus disease 2019

CI: Confidence interval

COR: $\quad$ Crude odds ratio

FMOH: Federal Ministry of Health

HCW: Healthcare worker

WHO: World Health Organization.

\section{Data Availability}

The data used to support the findings of this study are available from the corresponding author upon request.

\section{Additional Points}

As this area is not well studied, there was no any standard tool to investigate the HCPs' preventive practice. We developed the tool from different literature. We did not find adequate studies to compare and contrast, which makes our discussion shallow. Also, the findings of this study were based on HCPs' self-report. The self-report could be prone to social desirability and recall bias for preventive practice.

\section{Ethical Approval}

Ethical clearance was obtained from the Institutional Ethical Review Board of Debre Tabor University on 17 February 2020, Ref No. HSC/87/2012.

\section{Consent}

Informed consent was obtained from each healthcare provider after being informed all the purpose, benefits, risks, the confidentiality of the information, and the voluntary nature of participation in the study. Participants were informed that they have the right to discontinue at any point of the data collection.

\section{Conflicts of Interest}

The authors declare that there are no conflicts of interest.

\section{Authors' Contributions}

BAK and AKB conceived the study. All teams were involved in proposal development and coordinated the data collection activity. Statistical analysis was carried out by EAK, AA, and BAK. The manuscript was developed by BAK, AKB, EAK, and ASA. All authors critically reviewed, revised, and finally approved the manuscript.

\section{Acknowledgments}

The authors are very grateful to Debre Tabor University for the approval of the ethical clearance. The authors would like to thank North and South Gondar zonal health departments for their cooperation and support. They are also thankful to data collectors and supervisors and, most importantly, their study participants for their willingness to participate in the study.

\section{References}

[1] CDC, Novel Coronavirus, CDC, Wuhan City, China, 2019.

[2] WHO, Rolling Updates on Coronavirus Disease, WHO, Geneva, Switzerland, 2020.

[3] D. B. Jernigan, "Update: public health response to the coronavirus disease 2019 outbreak-United States, february 24, 2020," MMWR Morbidity and Mortality Weekly Report, vol. 69, no. 8, pp. 216-219, 2020.

[4] WHO, "Corona virus live updates at Newyork time," WHO, Geneva, Switzerland, 2020.

[5] WHO, "Mode of transmission of COVID-19. Implication for IPC precaution recommendations," WHO, Geneva, Switzerland, 2020.

[6] UNICEF, "COVID-19 emergency preparedness and response, wash and infection prevention and control in health facilities guidance note," UNICEF, New York, NY, USA, 2020.

[7] "Worldometer's," 2020, https://www.worldometers.info/ coronavirus/2020.

[8] Worled Economic Foruem, "Why sub-Saharan Africa needs a unique response to COVI-19," Worled Economic Foruem, Cologny, Switzerland, 2020.

[9] ECDC, "Infection prevention and control and preparedness for COVID-19 in health care settings," ECDC Technical Report, ECDC, Solna Sweden, 2020.

[10] C. Ağalar and D. Ö. Engin, "Protective measures for COVID19 for healthcare providers and laboratory personnel," Turkish Journal of Medical Science, vol. 50, no. SI-1, pp. 578-584, 2020.

[11] C. Houghton, P. Meskell, H. Delaney et al., "Barriers and facilitators to healthcare workers adherence with infection prevention and control (IPC) guidelines for respiratory infectious disease, a rapid qualitative evidence synthesi," The Cochrane Database of Systematic Reviews, vol. 4, no. 4, p. CD013582, 2020.

[12] AMN Helthcare Education Services, The Nurse's Role in Preventing Hospital Acquired Infections, AMN Helthcare Education Services, San Antonio, TX, USA, 2019.

[13] C. Parmeggiani, R. Abbate, and I. Angelillo, "Health care workers and healthcare associated infections: knowldge, attitude and behaviour in emergency departments in Italy," BMC Infectios Disease, vol. 10, no. 35, 2010.

[14] Y. Chen, X. Tong, J. Wang et al., "High SARS-CoV-2 antibody prevalence among healthcare workers exposed to COVID-19 patients," Journal of Infection, vol. 81, no. 3, pp. 420-426, 2020.

[15] M. Zhou, F. Tang, Y. Wang et al., "Knowledge, attitude and practice regarding COVID-19 among health care workers in Henan, China," Journal of Hospital Infection, vol. 105, no. 2, pp. 183-187, 2020. 
[16] R. Olum, G. Chekwech, G. Wekha, D. R. Nassozi, and F. Bongomin, "Coronavirus disease-2019: knowledge, attitude, and practices of health care workers at Makerere University Teaching Hospitals, Uganda," Frontiers in Public Health, vol. 8, p. 181, 2020.

[17] S. Halasey, "Shortage of personal protective equipment endangering health workers worldwide," WHO, Geneva, Switzerland, 2020.

[18] P. Planning, "Recommended guidance for extended use and limited reuse of N95 filtering facepiece respirators in healthcare settings," CDC, Washington, DC, USA, 2020.

[19] B. V. Shenal, L. J. Radonovich, J. Cheng, M. Hodgson, and B. S. Bender, "Discomfort and exertion associated with prolonged wear of respiratory protection in a health care setting," Journal of Occupational and Environmental Hygiene, vol. 9, no. 1, pp. 59-64, 2012.

[20] A. A. Chughtai, S. Stelzer-Braid, W. Rawlinson et al., "Contamination by respiratory viruses on outer surface of medical masks used by hospital healthcare workers," BMC Infectious Diseases, vol. 19, no. 1, p. 491, 2019.

[21] B.-L. Zhong, W. Luo, H.-M. Li et al., "Knowledge, attitudes, and practices towards COVID-19 among Chinese residents during the rapid rise period of the COVID-19 outbreak: a quick online cross-sectional survey," International Journal of Biological Sciences, vol. 16, no. 10, pp. 1745-1752, 2020.

[22] A. A. Azlan, M. R. Hamzah, T. J. Sern, S. H. Ayub, and E. Mohamad, "Public knowledge, attitudes and practices towards COVID-19: a cross-sectional study in Malaysia," PLoS One, vol. 15, no. 5, Article ID e0233668, 2020. 